\title{
Testing the coherence between occupational exposure limits for inhalation and their biological limit values with a generalized PBPK-model: The case of 2-propanol and acetone
}

\author{
Daan Huizer ${ }^{\mathrm{a}, \mathrm{b}, *}$, Mark A.J. Huijbregts ${ }^{\mathrm{a}}$, Joost G.M. van Rooij ${ }^{\mathrm{b}}$, Ad M.J. Ragas ${ }^{\mathrm{a}, \mathrm{c}}$

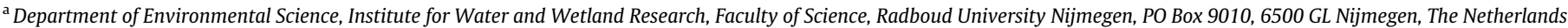 \\ ${ }^{\mathrm{b}}$ Caesar Consult Nijmegen, PO Box 31070, 6503 CB Nijmegen, The Netherlands \\ ${ }^{\text {' } S c h o o l ~ o f ~ S c i e n c e, ~ O p e n ~ U n i v e r s i t e i t, ~ P O ~ B o x ~ 2960, ~} 6401$ DL Heerlen, The Netherlands
}

\section{A R T I C L E I N F O}

Article history:

Received 22 November 2013

Available online $\mathrm{xxxx}$

\section{Keywords:}

Coherence

Occupational exposure limits

Biological limit values

PBPK model

2-Propanol

Acetone

Uncertainty

Variability

\begin{abstract}
A B S T R A C T
The coherence between occupational exposure limits (OELs) and their corresponding biological limit values (BLVs) was evaluated for 2-propanol and acetone. A generic human PBPK model was used to predict internal concentrations after inhalation exposure at the level of the OEL. The fraction of workers with predicted internal concentrations lower than the BLV, i.e. the 'false negatives', was taken as a measure for incoherence. The impact of variability and uncertainty in input parameters was separated by means of nested Monte Carlo simulation. Depending on the exposure scenario considered, the median fraction of the population for which the limit values were incoherent ranged from $2 \%$ to $45 \%$. Parameter importance analysis showed that body weight was the main factor contributing to interindividual variability in blood and urine concentrations and that the metabolic parameters $V_{\max }$ and $K_{\mathrm{m}}$ were the most important sources of uncertainty. This study demonstrates that the OELs and BLVs for 2-propanol and acetone are not fully coherent, i.e. enforcement of BLVs may result in OELs being violated. In order to assess the acceptability of this "incoherence", a maximum population fraction at risk of exceeding the OEL should be specified as well as a minimum level of certainty in predicting this fraction.
\end{abstract}

(c) 2014 Elsevier Inc. All rights reserved.

\section{Introduction}

Monitoring of workplace exposure to chemicals has become common practice in many countries (Angerer et al., 2011). In this context, occupational exposure limits (OELs) for air, such as threshold limit values (TLVs), Maximale Arbeitzplatz-Konzentration werten (MAK-werten) and derived no effect limits for workers (DNEL Worker), have been derived to control the health risk of chemical exposure in occupational settings. Additionally, biological limit values (BLVs) such as biological exposure indices (BEI) and Biologische Arbeitsstoff-Toleranzwerten (BAT) have been derived as reference values for biological media, such as blood and urine. Another approach in which external dose-based guidance values, such as the tolerable daily intake (TDI) or reference dose (RfD), are translated into so-called biomonitoring equivalent $(\mathrm{BE})$ values

\footnotetext{
* Corresponding author at: Department of Environmental Science, Faculty of Science, Radboud University Nijmegen, PO Box 9010, 6500 GL Nijmegen, The Netherlands. Fax: +31243653030.

E-mail addresses: d.huizer@science.ru.nl, daan.huizer@caesar-consult.nl (D. Huizer), m.huijbregts@science.ru.nl (M.A.J. Huijbregts), joost.vanrooij@caesar-consult.nl (J.G.M. van Rooij), a.ragas@science.ru.nl (A.M.J. Ragas).
}

is increasingly being used in the public health context, but so far not in occupational settings (Hays et al., 2007, 2008).

Although BLVs are currently still limited in number (derived for roughly 50 industrial chemicals), they strongly support and legitimize the application of biological monitoring as a method to measure and control human exposure to chemical substances (Angerer et al., 2007; Boogaard et al., 2011). Biological monitoring is embedded in EU regulations DIR 80/1107/EEC, DIR 89/391/EEC and DIR 98/24/EC.

BLVs are typically derived from OELs, based on an empirical relationship between external exposure levels and corresponding internal concentrations (ACGIH, 2001; Angerer et al., 2011). In these cases, enforcement of BLVs should guarantee that OELs are not being exceeded since these OELs were originally derived from experimental toxicological data (Thomas et al., 1996). A violation of an OEL may remain unnoticed when the BLV is not being exceeded at an exposure level that corresponds to the OEL. In the present study, an OEL and its corresponding BLV are therefore called coherent if the predicted internal concentration after exposure to the OEL equals or exceeds the BLV. False negatives, i.e. internal concentrations below the BLV after exposure at the level of the OEL, should be avoided. 
In recent years, several physiologically based pharmacokinetic (PBPK) models have been developed to support the derivation of BLVs (Adams et al., 2005; Bartels et al., 2012; Leung, 1992; Tardif et al., 2002; Truchon et al., 2013). However, coherence testing between BLVs and OELs with PBPK models has only been performed to a limited extent. Thomas et al. (1996) predicted the interindividual variability in the concentrations of chemicals in worker's exhaled breath and urine after exposure to OELs of 6 industrial chemicals. They compared the results with the corresponding BLVs. For the urinary metabolites of benzene, methyl chloroform and trichloroethylene, they found that $54-97 \%$ of the worker population is protected at the level of the BLV. However, Thomas et al. (1996) only considered interindividual variability in input parameters, and not uncertainty. It is important to distinguish these two sources of variation since they have different implications for policy makers. Whereas interindividual variability indicates the population fraction at risk and provides insight into the characteristics of susceptible individuals (Nestorov, 2001), uncertainty determines the reliability of model predictions (Cullen, 1998; Morgan and Henrion, 1990) and provides insight into the most efficient ways to improve model predictions, e.g. by more precise measurements (Nestorov, 2001; Ragas et al., 2009).

The aim of the present study was to test the coherence between the OELs and BLVs of two commonly used solvents, i.e. 2-propanol and acetone. The BLVs of these substances were originally derived from the OELs using linear regression between external and internal exposure levels. The PBPK model BioNormtox (Huizer et al., 2012) was used here to predict blood and urine concentrations after exposure to air concentrations at the level of the OEL. These predicted internal concentrations were then compared to the corresponding BLVs, resulting in a measure of coherence. The impact of uncertainty and interindividual variability was quantified separately by means of nested Monte Carlo simulation.

\section{Material and methods}

\subsection{BioNormtox model}

\subsubsection{Outline}

We used the PBPK model BioNormtox, an extended version of the IndusChemFate model as previously described by Huizer et al. (2012), to assess the coherence between OELs and BLVs for 2-propanol and acetone. In the same publication, it was shown that simulations for these substances were in line with experimental human data. The model is briefly described here; a more detailed model description can be found in Huizer et al. (2012). The human body is represented in BioNormtox by 10 main compartments: (1) lung, (2) heart, (3) brain, (4) skin, (5) fat, (6) muscles, (7) bone, (8) stomach and intestines, (9) liver and (10) kidney (Fig. 1). The lungs are subdivided into bronchioles, mucosa, and alveolae to model the washin-washout effect of solvent vapors in the respiratory tract, following Mork et al. (2009) and Mork and Johanson (2006). Partitioning between different compartments is based on a QSAR for tissue:water partitioning, originally developed by Hendriks et al. (2005). Metabolism (Michaelis-Menten kinetics) is based on the metabolic parameters $V_{\max }$ (maximum velocity of metabolism) and $K_{\mathrm{m}}$ (Michaelis-Menten constant).

\subsubsection{Parameter uncertainty and variability}

Variation in the model output was quantified as interindividual variability and uncertainty separately, based on assigned parameter distributions by Huizer et al. (2012). Interindividual variability for resting conditions was described using literature-based distributions for all physiological parameters (Table 1). Lognormal distributions were used for continuous physiological parameters, whereas fractional parameters were assigned betaPERT distributions.

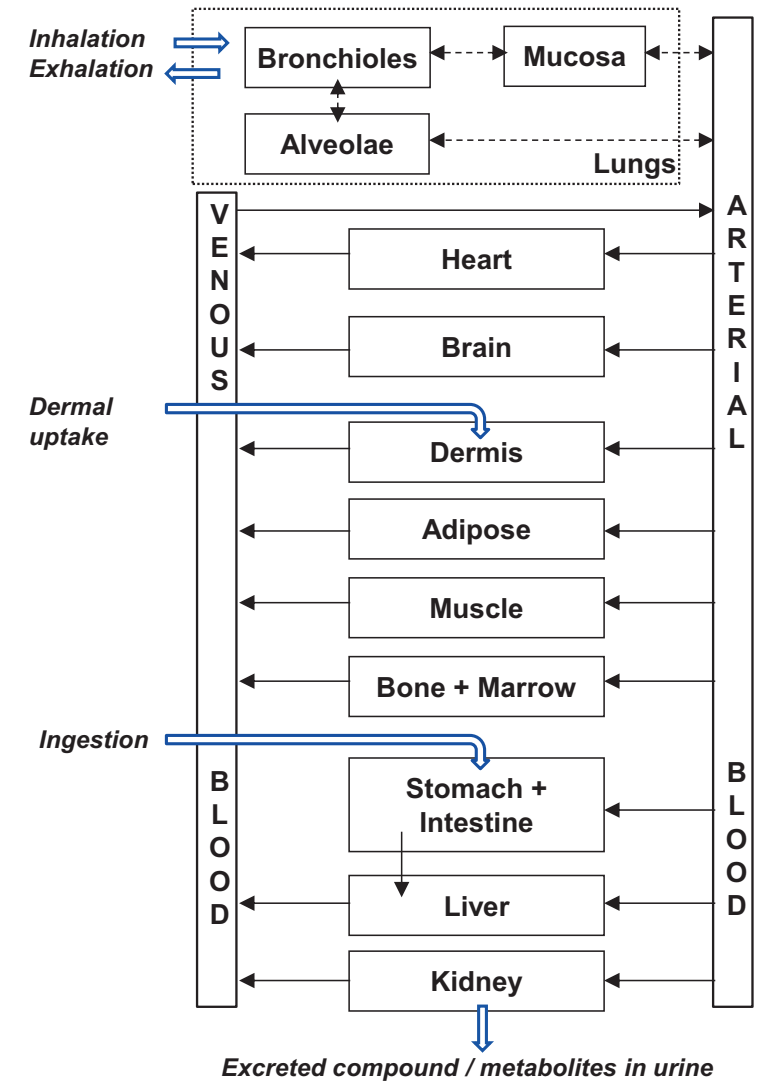

Fig. 1. Structure of the BioNormtox model. The continuous lines represent blood flows between tissues, whereas the dashed lines represent the exchange of the parent compound or metabolites between the respiratory tract and the arterial blood.

Uncertainty distributions were derived for physico-chemical parameters of 2-propanol and acetone (lognormal) and chemical affinity intercepts and exponents (betaPERT). The metabolic parameters $K_{\mathrm{m}}$ and $V_{\text {max }}$ were considered as variable and uncertain, since variation in these parameter values may originate from both interindividual variability (i.e., differences in enzymatic activity between individuals) and uncertainty (i.e., differences in experimental design and measurement uncertainty). For the present study, all parameter distributions related to physico-chemical properties and biotransformation of 2-propanol and acetone were taken from Huizer et al. (2012) (Tables A-1 and A-2).

\subsubsection{Model adjustments}

In comparison to the original model, two major adjustments were made: (1) renal excretion was now modeled as a process that is described by the water soluble fraction of the substance in renal blood and the production rate of urine, i.e. replacing the original arbitrary cut-off value for glomerular filtration (Eq. A-1), and (2) the parameter distributions for human physiology were changed to conditions that correspond to light work in order to simulate more realistic workplace scenarios (see Section 2.2). A summary of the parameter distributions for human physiology in rest and at light work is presented in Table 1 . The derivation of parameter distributions for light work is described in more detail below.

\subsection{Human physiology}

Increased exercise may lead to changes in tissue perfusion and ventilation characteristics. These changes originate from three main physiological principles: (1) increase of cardiac output, (2) 
Table 1

Variability distributions for physiological parameters in rest and during light work as used in the model simulations (see also Huizer et al., 2012 for further details).

\begin{tabular}{|c|c|c|c|c|c|}
\hline Description of variable & Units & Distribution type & At rest ${ }^{\mathrm{a}}$ & At light work $^{\mathrm{a}}$ & Multiplier (rest to exercise) \\
\hline Alveolar ventilation $^{b}$ & $\mathrm{l} / \mathrm{h}$ & Lognormal & $511(1.27)$ & $1334(1.14)$ & Not applicable \\
\hline Cardiac output $^{\mathrm{b}}$ & $1 / \mathrm{h}$ & Lognormal & $390(1.24)$ & $591(1.11)$ & Not applicable \\
\hline Body weight & $\mathrm{kg}$ & Lognormal & $71.4(1.24)$ & $71.4(1.24)^{d}$ & Not applicable \\
\hline Thickness of epidermis & $\mathrm{cm}$ & Lognormal & $0.0084(1.22)$ & $0.0084(1.22)^{\mathrm{d}}$ & Not applicable \\
\hline Thickness of stratum corneum & $\mathrm{cm}$ & Lognormal & $0.0015(1.37)$ & $0.0015(1.37)^{d}$ & Not applicable \\
\hline Volume of produced urine & l/h & Lognormal & $0.091(1.51)^{c}$ & $0.091(1.51)^{d}$ & Not applicable \\
\hline Arterial fraction & Unitless & BetaPERT & $0.251(0.167,0.376)$ & $0.251(0.167,0.376)^{d}$ & Not applicable \\
\hline \multicolumn{6}{|l|}{ Cardiac output fractions to tissues } \\
\hline Adipose tissue & Unitless & BetaPERT & $0.061(0.020,0.184)$ & $0.104(0.035,0.313)$ & 1.7 \\
\hline Brain & Unitless & BetaPERT & $0.117(0.078,0.176)$ & $0.07(0.047,0.105)$ & 0.6 \\
\hline Heart & Unitless & BetaPERT & $0.056(0.037,0.083)$ & $0.056(0.037,0.083)$ & 1 \\
\hline Kidneys & Unitless & BetaPERT & $0.177(0.118,0.265)$ & $0.088(0.059,0.133)$ & 0.5 \\
\hline Liver arterial & Unitless & BetaPERT & $0.068(0.046,0.103)$ & $0.034(0.023,0.051)$ & 0.5 \\
\hline Liver venous & Unitless & BetaPERT & $0.189(0.126,0.283)$ & $0.094(0.063,0.141)$ & 0.5 \\
\hline Lung & Unitless & BetaPERT & $0.034(0.023,0.051)$ & $0.034(0.023,0.051)$ & 1 \\
\hline Muscle & Unitless & BetaPERT & $0.142(0.095,0.214)$ & $0.313(0.209,0.470)$ & 2.2 \\
\hline Skeleton (bone) & Unitless & BetaPERT & $0.115(0.077,0.173)$ & $0.115(0.077,0.173)$ & 1 \\
\hline Skin & Unitless & BetaPERT & $0.059(0.039,0.089)$ & $0.107(0.071,0.160)$ & 1.8 \\
\hline \multicolumn{6}{|c|}{ Fractional transfer coefficients for washin-washout module of model } \\
\hline Mucosa to arterial blood & Unitless & Beta & $25.26,8.88(0,1)$ & $25.26,8.88(0,1)^{d}$ & Not applicable \\
\hline Bronchioles to mucosa & Unitless & Beta & $29.67,3.67(0,1)$ & $29.67,3.67(0,1)^{d}$ & Not applicable \\
\hline
\end{tabular}

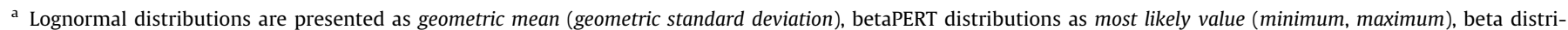
butions as alpha, beta (minimum, maximum).

b Alveolar ventilation and cardiac output were assumed to be correlated with a correlation coefficient of 0.8 (Levitzky, 2003 ).

c Includes individuals from general population in all states of exercise, based on Raman et al. (2004).

d Unchanged at light work compared to situation in rest.

increase of alveolar ventilation, and (3) change of distribution of the cardiac output over tissues (Fiserova-Bergerova, 1983). Corresponding parameter distributions for human physiology in the BioNormtox model were adjusted to represent light work. In line with Astrand (1983), we defined light work as a workload of 35-50 W. This corresponds to daily scenarios for most occupational settings, including work related to cleaning, construction, farming, industrial processing, machine operating and painting (Ainsworth et al., 2011).

\subsubsection{Cardiac output and alveolar ventilation}

Data from Astrand (1983) were used to derive a lognormal distribution that describes human variability of cardiac output during light work. Similarly, a distribution for the description of variability in alveolar ventilation was derived based on data by Astrand (1983).

\subsubsection{Fractional blood flows}

For the perfusion of tissues during light work, the parameter distributions for fractional blood flows (as fraction of the total cardiac output) in rest were used as a starting point, since these distributions were well established based on a large number of empirical data by Huizer et al. (2012). Deterministic multipliers were used to convert these distributions from rest to light work (Eq. (1)).

Eq. (1) - principle of scaling of fractional blood flows to tissues in rest to light work.

$\mathrm{QC}_{\text {tissue light work }}=\mathrm{QC}_{\text {tissue rest }} * \mathrm{R}_{\text {tissue }}$

where $\mathrm{QC}_{\text {tissue light work }}$ is the fractional blood flow to a tissue at light exercise (\% of cardiac output), $\mathrm{QC}_{\text {tissue rest }}$ the fractional blood at rest (\% of cardiac output) and $\mathrm{R}_{\text {tissue }}$ the multiplier per tissue type (dimensionless). The multipliers were calculated as the ratio between typical values for fractional blood flows to tissues at rest and during light work. The mean values reported in Huizer et al. (2012) were used as typical values at rest. Typical values during light work were derived from multiple data sources and then aggregated per tissue type (Table A-3).

\subsubsection{Other physiological parameters}

The original distribution representing the volume of produced urine was left unchanged as it includes data from individuals both in rest and during light work (Raman et al., 2004). The fractional transfer coefficients for the washin-washout module in the model were also left unchanged, since these are related to the alveolar ventilation and are therefore scaled via this parameter (Mork et al., 2009).

\subsection{Coherence testing}

The coherence between OELs and BLVs was determined by comparing the simulated blood or urine concentrations after inhalation exposure at the level of the OEL for the defined exposure duration to the corresponding BLV of the parent chemical or its metabolite. We calculated the fraction of 'false negatives' in the worker population, i.e. the fraction of workers with predicted internal concentrations below the BLV after exposure to the OEL. These false negatives indicate cases where a violation of the OEL does not necessarily result in a violation of the BLV. The larger the fraction of 'false negatives', the less coherence is demonstrated.

Exposure to acetone and 2-propanol via inhalation was simulated with the BioNormtox model for the following scenarios (Table 2):

(1) A scenario of $8 \mathrm{~h}$ constant exposure to acetone at the OEL (500 ppm in air) during light exercise, to test the coherence with the BLV of acetone in urine.

(2) A scenario of $8 \mathrm{~h}$ constant exposure to 2-propanol at the OEL (200 ppm in air) during light exercise, to test the coherence with the BLVs of acetone in blood.

(3) A scenario of $8 \mathrm{~h}$ constant exposure to 2-propanol at the OEL (200 ppm in air) during light exercise, to test the coherence with the BLVs of acetone in urine. 
Table 2

Occupational exposure limits (OELs) and Biological limit values (BLVs) for acetone and 2-propanol.

\begin{tabular}{|c|c|c|c|c|c|c|c|c|c|}
\hline \multicolumn{4}{|c|}{ Occupational exposure limit } & \multicolumn{6}{|c|}{ Biological limit value } \\
\hline \multicolumn{4}{|c|}{ Parent compound in working atmosphere } & \multicolumn{3}{|c|}{ Acetone in blood } & \multicolumn{3}{|c|}{ Acetone in urine } \\
\hline & $(\mathrm{ppm})$ & Time $(h)^{*}$ & Reference & $(\mathrm{mg} / \mathrm{l})$ & Time $(\mathrm{h})$ & Reference & $(\mathrm{mg} / \mathrm{l})$ & Time $(\mathrm{h})$ & Reference \\
\hline \multirow[t]{2}{*}{ Acetone } & $500^{* *}$ & 8 & MAK (1993) & - & - & - & 80 & 8 & BAT (1995) \\
\hline & & & TLV (1996) & - & - & - & 50 & 8 & BEI (1999) \\
\hline \multirow[t]{2}{*}{ 2-Propanol } & $200^{* * * *}$ & 8 & MAK (1996) & 25 & 8 & BAT, 2009 & 25 & 8 & BAT (2009) \\
\hline & & & TLV (2003) & - & - & - & 40 & $5 \times 8$ & BEI (2006) \\
\hline
\end{tabular}

* From onset of exposure $(t=0 \mathrm{~h})$.

** $1188 \mathrm{mg} / \mathrm{m}^{3}$ acetone.

**** $490 \mathrm{mg} / \mathrm{m}^{3}$ 2-propanol.

(4) A scenario of 5 times $8 \mathrm{~h}$ exposure to 2-propanol at the OEL (200 ppm in air) during light exercise, to test the coherence with the BLV of 2-propanol in urine at the end of the workweek $(t=104 \mathrm{~h})$.

To account for the endogenous urinary level of acetone in the general population, which is implicitly accounted for in the BLV, the endogenous urinary concentration was added to the predicted urinary concentration of acetone. This endogenous urinary concentration was sampled from a lognormal distribution, based on literature data (Table A-5) (Ghittori et al., 1996; Kawai et al., 1992; Pezzagno et al., 1986; Satoh et al., 1995; Wang et al., 1994; Wigaeus et al., 1981).

\subsection{Nested Monte Carlo simulation}

Interindividual variability in physiological parameters and true uncertainty in physico-chemical parameters were separated by means of nested Monte Carlo simulation using Crystal Ball software (Oracle, version 11). For each scenario in Table 2, a nested Monte Carlo simulation was performed based on a randomly selected set of 100 values from each of the variable parameters and a randomly selected set of 100 values from each of the uncertain parameters. In the analysis, the uncertain parameter sets were fixed one by one and the 100 variability values were iterated, running the BioNormtox model after each iteration. This process was repeated for each uncertainty set, resulting in 100 population distributions where each distribution reflects the interindividual variability within a population. The results of the simulations were then ranked, based on the median values of the population distributions to reveal the variance between these populations as a measure of uncertainty. Finally, the 10th, 50th and 90th population distributions were plotted as cumulative probability plots in which variability and uncertainty are both represented.

\subsection{Importance analysis}

The relative contribution of the input parameters to the variability and uncertainty of the output parameters was calculated by means of a modified Spearman rank correlation method as described by Huizer et al. (2012). First, the correlation coefficients were recalculated to correct for correlation between the cardiac output and alveolar ventilation by using partial Spearman rank correlation coefficients (Hamby, 1994). Second, the overall importance of parameters that were defined as both variable and uncertain, i.e. the metabolic parameters $V_{\max }$ and $K_{\mathrm{m}}$, was separated into an uncertainty and a variability component (Huizer et al., 2012). The importance analysis was performed for each of the scenarios in Table 2.

\section{Results}

\subsection{Coherence between OELs and BLVs for acetone and 2-propanol}

The cumulative probability plots in Fig. 2 display uncertainty and variability in predicted concentrations of acetone in blood and urine after inhalation exposure to acetone and 2-propanol in accordance with the scenarios in Table 2 . In each of these plots, three curves are displayed, representing three of the 100 simulated potential populations. They correspond with the 10th, 50th and 90th uncertainty percentiles of the simulation results for each exposure scenario. Variance within one cumulative distribution, reflected by the slope, represents interindividual variability within the population. The variation between the cumulative distributions, reflecting different populations, represents uncertainty (illustrated with the arrows in Fig. 2c). In the same figures, the applicable BLV is depicted.

Coherence between the OEL and the corresponding BLV is demonstrated when inhalation exposure at the level of the OEL does not result in concentrations in blood or urine below the BLV. The level of coherence was specified by quantification of the predicted fraction of 'false negatives', i.e. individuals with predicted internal concentrations below the BLV after exposure to the OEL, in the worker population (variability) and the corresponding probability level of this prediction (uncertainty). Fig. 2 shows that there is a $50 \%$ chance that the fraction of false negatives is $2 \%$ of the population in comparison with the American BLV (BEI) for acetone in urine, whereas there is $10 \%$ chance that this fraction is $10 \%$. In case the German BLV (BAT) for the same scenario is considered, there is a $50 \%$ chance that population fraction of false negatives is $45 \%$, whereas there is $10 \%$ chance that this is as much as $75 \%$.

In Table 3 the coherence between the OELs and the BLVs for acetone and 2-propanol is presented for each simulated scenario. This coherence is expressed as the predicted fraction of false negatives in the worker population for three different probability levels (i.e., the 10 th, 50th and 90th percentile). Table 3 shows that the median fraction of false negatives in the worker population ranges from $2 \%$ to $45 \%$ over the four different scenarios. The 10 th percentile of the false negatives in the worker population ranges from $10 \%$ to $93 \%$, while the 90 th percentile ranges from $0 \%$ to $20 \%$. The largest uncertainty in the calculated fraction is found for 2-propanol in the end of workweek scenario.

\subsection{Parameter importance}

Fig. 3 shows the results of the parameter importance analysis. For all scenarios, variability plays a major role in the variation in blood and urine concentrations (60-80\% of the total variation), mainly as a result of the human physiological parameters body weight and alveolar ventilation. Uncertainty and variability in the metabolic parameters $V_{\max }$ and $K_{\mathrm{m}}$ are also an important source 
(a)

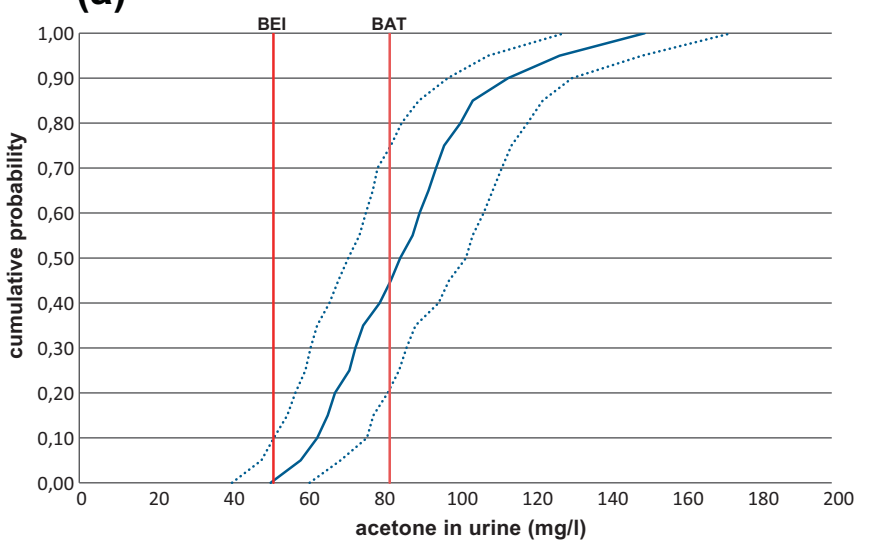

(c)

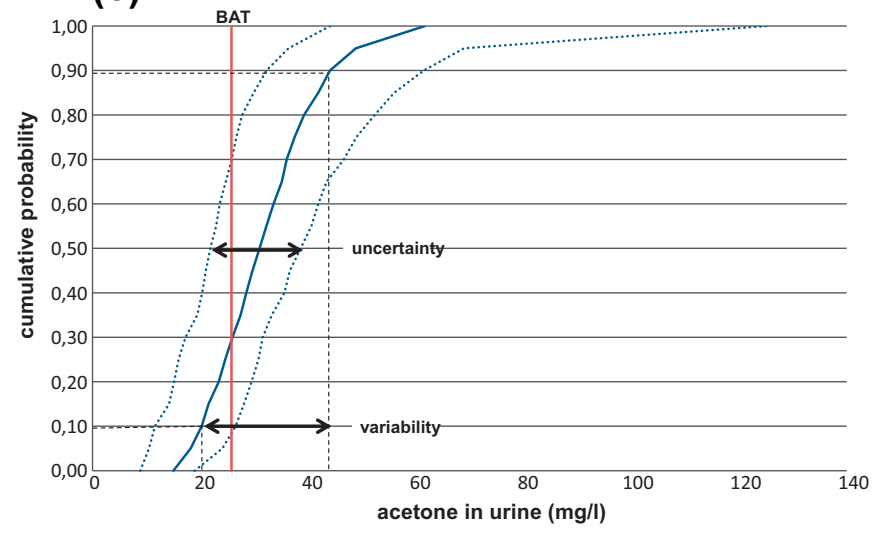

(b)

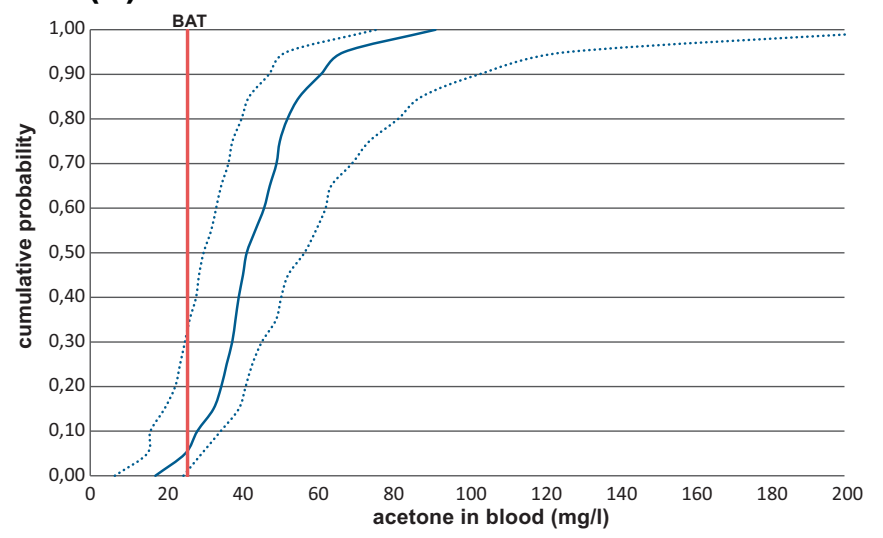

(d)

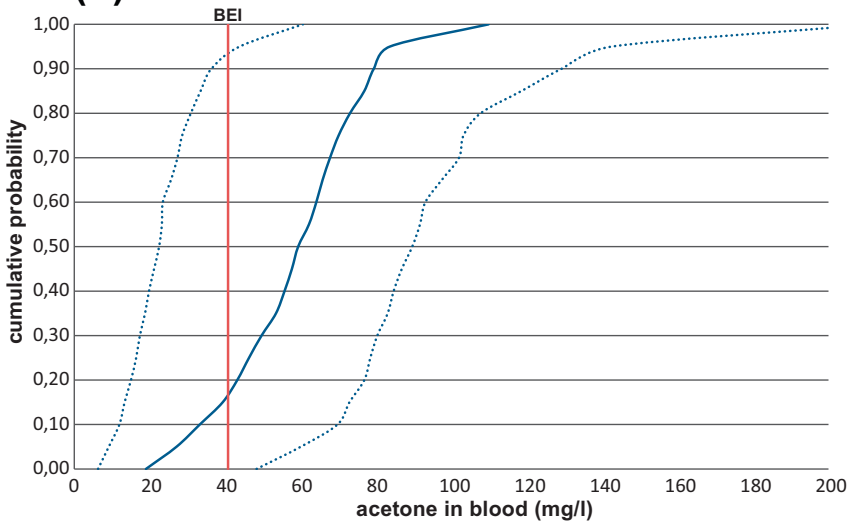

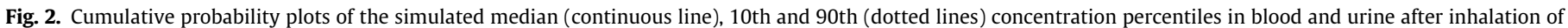
acetone (2a) and 2-propanol (2b, c, d) at the level of the OEL for $8 \mathrm{~h}$ during light exercise. Corresponding BLVs for each scenario are also displayed.

Table 3

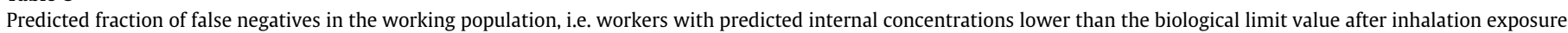
at the level of the OEL, as a measure of incoherence between limit values for acetone and 2-propanol as simulated with the BioNormtox model in four exposure scenarios.

\begin{tabular}{|c|c|c|c|c|}
\hline \multicolumn{3}{|c|}{ Exposure scenario } & \multirow[t]{2}{*}{ Biological limit value } & \multirow{2}{*}{$\begin{array}{l}\text { Predicted population fraction of false negatives } \\
\text { (incoherence) in \% (median value } \\
\text { with p10-p90 uncertainty range) }\end{array}$} \\
\hline No. & External & Internal & & \\
\hline \multirow[t]{2}{*}{1} & Acetone $(500 \mathrm{ppm}-8 \mathrm{~h})$ & Acetone in urine (end-of-workday) & BAT (D): $80 \mathrm{mg} / \mathrm{l}$ & $45 \%(20-75)$ \\
\hline & & & BEI (US): 50 mg/l & $2 \%(0-10)$ \\
\hline 2 & 2-Propanol (200 ppm - 8 h) & Acetone in blood (end-of-workday) & BAT (D): $25 \mathrm{mg} / \mathrm{l}$ & $5 \%(2-32)$ \\
\hline 3 & 2-Propanol (200 ppm - 8 h) & Acetone in urine (end-of workday) & BAT (D): $25 \mathrm{mg} / \mathrm{l}$ & $28 \%(8-67)$ \\
\hline 4 & 2-Propanol (200 ppm - $5 \times 8 \mathrm{~h}$ ) & Acetone in urine (end-of-workweek) & BEI (US): $40 \mathrm{mg} / \mathrm{l}$ & $17 \%(0-93)$ \\
\hline
\end{tabular}

of variation (10-30\% of the total variation), in particular for the 2 propanol scenarios. Other parameters that have a relatively large influence on the simulated blood and urine concentrations are parameters that are related to the QSAR for tissue partitioning, expressed as $K_{\mathrm{tw}}$ (lipid, protein and water fractions of tissues and their corresponding affinity exponents and intercepts) and $K_{\mathrm{ow}}$.

\section{Discussion}

First, model parameterizations and assumptions that may have influenced the results are discussed. Next, the coherence between the OELs and BLVs for 2-propanol and acetone is discussed. Finally, possible implications for policy makers are outlined.

\subsection{Influence of parameterization and assumptions}

To support the comparison between OELs and BLVs, the majority of the model parameters related to human physiology was scaled from rest to light work. As the availability of empirical human physiological data on fractional blood flows to tissues during light work was limited, the parameters distributions established for rest conditions were extrapolated to light work, using so called multipliers. This way, the robustness of the parameterization in rest as derived by Huizer et al. (2012) was maintained. However, the absence of detailed information about tissue lumping (i.e., the aggregation of similar tissues into groups) in multiple literature sources (Table A-3), as well as the lack of a specific definition of 'light work' may have influenced the final parameterization of the model. However, a comparison with data from FiserovaBergerova (1983) (Table A-4) indicates that the reported blood flows to relevant organs, such as the muscles, kidneys and liver, during light work are in line with the parameterization of the BioNormtox model.

Additional model simulations (Figs. 4 and A-1) show that the scaling of physiological parameters from rest to light work has a significant influence on the predicted blood and urine concentration 

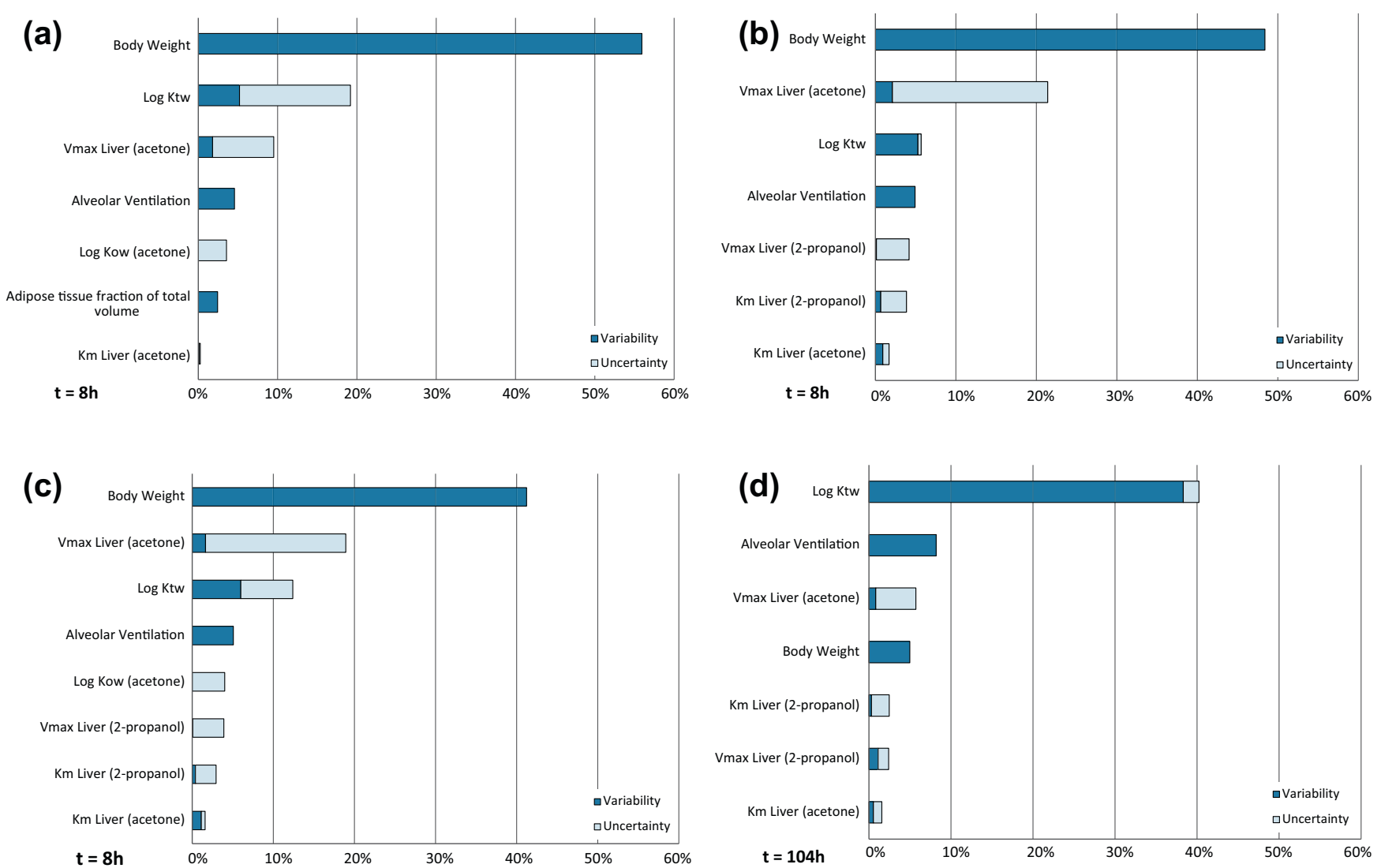

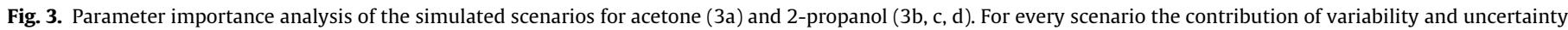
to the total variation is presented per parameter.

of acetone; the internal concentrations decrease with decreasing exercise levels. Mork and Johanson (2006) reported similar results for acetone based on experimental human data and model simulations. Schaller and Triebig (2002) also report an increase of the acetone concentration in urine with increasing activity. These results illustrate that the level of physical exercise significantly influences the relationship between external and internal exposure concentrations. For our exposure scenarios, we would have found lower coherence levels at lower exercise levels. To put it in another way: at lower exercise levels there is a higher risk of the OEL being exceeded while the BLV is not being exceeded.

Another factor that may have influenced the model predictions is the parameterization of renal excretion. Urinary excretion in PBPK models for 2-propanol and acetone has generally been parameterized as a first-order process (Clewell et al., 2001) or based on partitioning (Kumagai and Matsunaga, 1995). Mork and Johanson (2006) neglected this route entirely in their acetone model, stating that urinary excretion is not a significant elimination route for this substance. In our model renal excretion depends on the water soluble fraction of the substance in renal blood and the production rate of urine, replacing the arbitrary cut-off value for glomerular filtration in the Huizer et al. (2012) model. This modification also allowed a probabilistic approach of the urinary excretion through the derived parameter distributions involved. This results in urinary excretion levels around $1 \%$ of the total dose for both 2-propanol and acetone, which is in line with the published models and the background documentation of the BLVs (ACGIH, 2001; DFG, 2012).

Finally, possible covariation between metabolic parameters $V_{\max }$ and $K_{\mathrm{m}}$ was not considered in our analyses, due to a lack of quantitative information on the exact correlation between these two parameters. However, as covariation is expected to be relevant mainly for the variability components of $V_{\max }$ and $K_{\mathrm{m}}$, based on interindividual variation in the activity of metabolic enzymes (Dorne et al., 2004; Martinez et al., 2010), and the importance of uncertainty was considerably higher in comparison with interindividual variability for these parameters in our analyses, it is expected that ignoring the possible covariation has influenced the results only to a limited extend.

\subsection{Coherence between exposure limits for 2-propanol and acetone}

In this study, four different exposure scenarios were assessed. These scenarios include variation in exposure concentration, exposure duration, type of biological medium (blood and urine) and metabolic phase (parent and first metabolite).

Based on our coherence test, we found that the BLVs for 2-propanol and acetone are likely (i.e., with $50 \%$ certainty) to be coherent with their OEL for the majority of the working population (55-98\%). Our results are comparable to those of Thomas et al. (1996), who predicted protected population fractions ranging from $54 \%$ to $97 \%$ after exposure to benzene, methyl chloroform and trichloroethylene. However, Thomas et al. (1996) only included interindividual variability in input parameters, and not uncertainty. Our approach has the advantage that the probability of the predictions can also be specified.

For a further interpretation of our results, it is important to understand the scientific basis underlying the BLVs, i.e. the BAT and BEI values for acetone and 2-propanol. German BAT values are limit values which should not be exceeded, while the BEI values are advisory levels that may be exceeded by a fraction of the population. However, this is in most cases not explicitly defined 


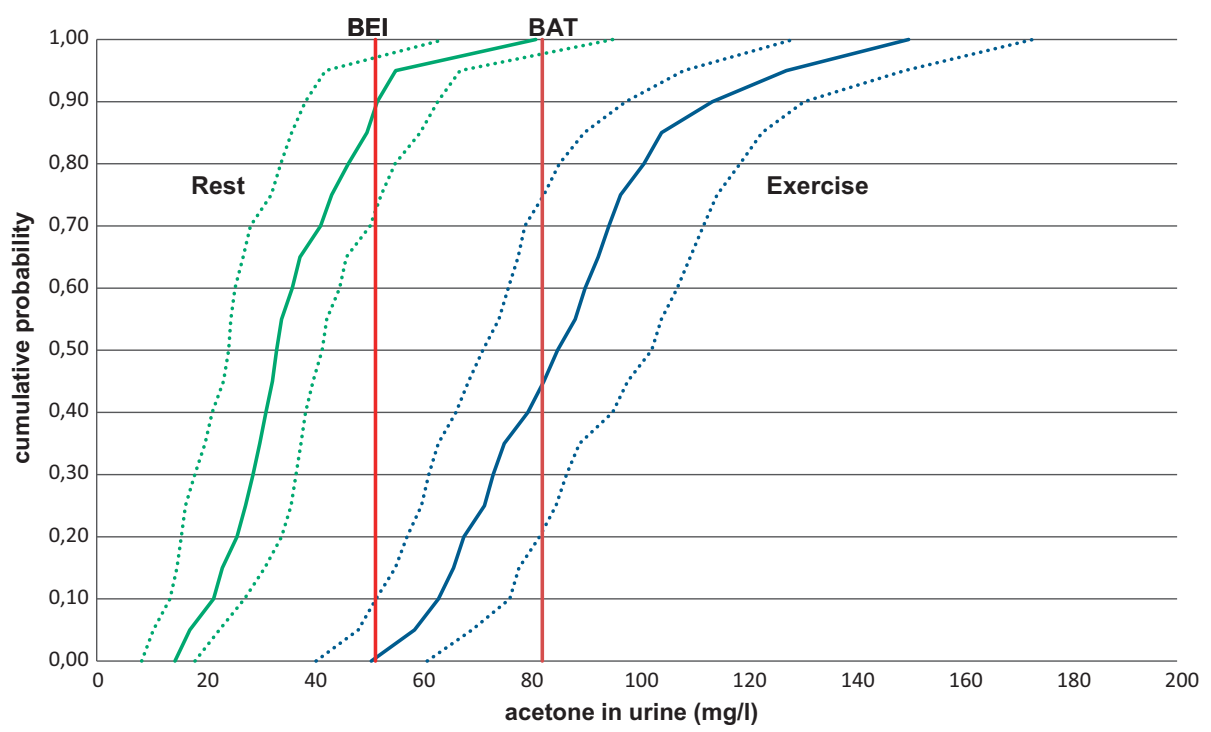

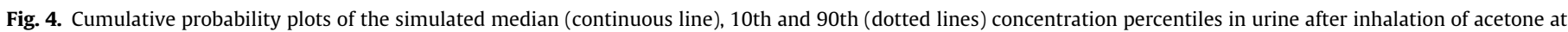
the level of the OEL for $8 \mathrm{~h}$ in rest and during light exercise. Corresponding BLVs for this scenario are also displayed.

(Morgan and Schaller, 1999). In the case of acetone and 2-propanol, both the BEI and BAT values have been established based on a relationship between external (inhalation) and internal (urine) exposure. Nevertheless, the BLVs differ substantially, i.e. 50 for the BEI vs. $80 \mathrm{mg} / \mathrm{l}$ urine for the BAT. This is most likely a result of differences in the interpretation of the assessed (exposure) data, as is illustrated in the background documentation of the BAT for acetone in which a range of potential limit values from 50 to $80 \mathrm{mg} /$ 1 urine is suggested. As a consequence of the arbitrary choice for $80 \mathrm{mg} / \mathrm{l}$ as a suitable BAT value, our results show a lower coherence level for this BAT value than for the BEI value.

The results of the parameter importance analyses provide insight into the main factors contributing to the variability and uncertainty in the model predictions. The physiological parameters body weight and alveolar ventilation and the metabolic parameters $V_{\max }$ and $K_{\mathrm{m}}$ turn out to be the most important sources of variation. Body weight is by far the most dominant parameter, as the volume of all model compartments is related to body weight. Internal concentrations tend to decrease with increasing body weight, implying that for individuals with a large body weight there is a higher chance of incoherence between the OEL and the BLV. The $V_{\max }$ for acetone is an important source of uncertainty, pointing towards acetone metabolism as an important elimination route.

Partitioning parameters become more important in scenarios in which urinary concentrations are predicted, which can be explained by the fact that only the dissolved fraction of acetone in blood is being excreted into urine.

\subsection{Practical implications}

The present study shows that OELs and BLVs of 2-propanol and acetone are not fully coherent, i.e. enforcement of BLVs does not always guarantee that OELs are being met. These OELs were originally derived from toxicity data and are therefore more directly linked to possible adverse health effects than BLVs. However, it should be stressed that incoherence does not necessarily imply adverse health effects. It is generally acknowledged that internal exposure is a better dose metric for toxicity than external exposure because the dose at the receptor site is an important driver of toxicity. Assuming equal receptor sensitivity, the value of the NOEL will mainly be determined by those individuals that reach relatively high internal concentrations under similar external exposure conditions. In our case, this would typically be the lightweight people with a high exercise level. Our study showed that heavy people with a low level of exercise have the highest risk of incoherence. This indicates that sensitivity is inversely related to incoherence, i.e. individuals with a high risk of incoherence (i.e., heavy persons with a low exercise level) will be sufficiently protected at the level of the OEL. Reasoning along these lines, a coherence level of 50\% would provide sufficient protection for the entire population.

The definition of coherence that we used in our case study is applicable only to cases where the OEL is derived from toxicity data and the BLV is subsequently derived from the OEL, e.g., based on an empirical relationship between external and internal concentrations. This definition of coherence changes if the BLV would be established directly based on toxicity data and the OEL would subsequently be derived from the BLV. In that case, the OEL and BLV are coherent only if the BLV is not being exceeded at the exposure level that corresponds to the OEL. In the context of risk assessment, coherence testing based on experimentally derived BLVs is preferred over BLVs that are derived from OELs based on the empirical relationship between external and internal concentrations. However, in practice data limitations may constrain this approach.

The large impact of exercise levels on the coherence of OELs and BLVs shows that OELs should not only consist of a concentration limit, but also include a specification of the exercise level for which it applies. Although it is generally recognized that the exercise levels influence internal concentrations, e.g. Astrand (1983) and Safe Work Australia (2012), specifications of exercise levels are typically limited to broad classifications such as "light work", "moderate work" and "heavy work"! A more detailed specification of these reference exercise levels for occupational exposure standards will support the safe application of OELs and BLVs under varying working conditions.

Our results show that uncertainty in the predicted concentrations is mainly caused by uncertainty in metabolic parameters (i.e. $V_{\max }$ and $K_{\mathrm{m}}$ of 2-propanol and acetone) and partitioning parameters (i.e. $K_{\mathrm{tw}}$ ). If uncertainty is to be reduced, research should focus on a more accurate determination of these metabolic and partitioning parameters. However, the first step is to determine whether reduction of uncertainty - and thus additional research - is desirable or necessary. This requires the specification of a minimum level of certainty for the predicted coherence level of 
the worker population, e.g. a 90\% probability level that the limit values are coherent for $50 \%$ of the worker population. In the present situation in which BLVs are based on experimentally derived OELs, regulatory authorities have two options if a coherence test shows that the combined levels of certainty and coherence level cannot be realized, i.e. either lower the BLV until sufficient coherence is realized or initiate new research to reduce uncertainty.

A major step forward in the reduction of incoherence would be the derivation of BLVs with PBPK modeling, analogous to the BE approach of Hays et al. (2008), in which the maximum allowed population fraction at risk and the level of certainty is defined upfront.

\section{Conflict of interest}

The authors declare that there are no conflicts of interest.

\section{Appendix A. Supplementary data}

Supplementary data associated with this article can be found, in the online version, at http://dx.doi.org/10.1016/j.yrtph.2014. 05.004.

\section{References}

ACGIH, 2001. Documentation of the threshold limit values for biological exposure indices. In: ACGIH (Ed.), Documentation of the Threshold Limit Values and Biological Exposure Indices, seventh ed., Cincinnati.

Adams, J.C. et al., 2005. A physiologically based toxicokinetic model of inhalation exposure to xylenes in Caucasian men. Regul. Toxicol. Pharmacol. 43, 203-214. Ainsworth, B.E. et al., 2011. 2011 Compendium of Physical Activities: a second update of codes and MET values. Med. Sci. Sports Exerc. 43, 1575-1581.

Angerer, J. et al., 2007. Human biomonitoring: state of the art. Int. J. Hyg. Environ. Health 210, 201-228.

Angerer, J. et al., 2011. Human biomonitoring assessment values: approaches and data requirements. Int. J. Hyg. Environ. Health 214, 348-360.

Astrand, I., 1983. Effect of physical exercise on uptake, distribution and elimination of vapours in man. In: Fiserova-Bergerova, V. (Ed.), Modelling of Inhalation Exposure to Vapours: Uptake, Distribution and Elimination, vol. 2. CRC Boca Raton, Florida.

Bartels, M. et al., 2012. Development of PK- and PBPK-based modeling tools for derivation of biomonitoring guidance values. Comput. Methods Programs Biomed. 108, 773-788.

Boogaard, P.J. et al., 2011. Human biomonitoring as a pragmatic tool to support health risk management of chemicals - examples under the EU REACH programme. Regul. Toxicol. Pharmacol. 59, 125-132.

Clewell 3rd, H.J. et al., 2001. Development of a physiologically based pharmacokinetic model of isopropanol and its metabolite acetone. Toxicol. Sci. 63, 160-172.

Cullen, A.C., Frey, H.C., 1998. Probabilistic Techniques in Exposure Assessment. A Handbook for Dealing with Variability in Uncertainty in Models and Inputs. Plenum Press, New York, USA.

DFG, D.F., 2012. MAK Values 2012. List of MAK and BAT Values 2012. Wiley-VCH Verlag GmbH \& Co. KGaA, pp. 1-298.

Dorne, J.L., Walton, K., Renwick, A.G., 2004. Human variability for metabolic pathways with limited data (CYP2A6, CYP2C9, CYP2E1, ADH, esterases, glycine and sulphate conjugation). Food Chem. Toxicol. 42, 397-421.

Fiserova-Bergerova, V., 1983. Modeling of Inhalation Exposure to Vapors; Uptake, Distribution, and Elimination. CRC Press, Boca Raton, Florida.

Ghittori, S. et al., 1996. Acetone in urine as biological index of occupational exposure to isopropyl alcohol. Ind. Health 34, 409-414.
Hamby, D.M., 1994. A review of techniques for parameter sensitivity analysis of environmental models. Environ. Monit. Assess. 32, 135-154.

Hays, S.M. et al., 2007. Biomonitoring equivalents: a screening approach for interpreting biomonitoring results from a public health risk perspective. Regul. Toxicol. Pharmacol. 47, 96-109.

Hays, S.M. et al., 2008. Guidelines for the derivation of biomonitoring equivalents: report from the biomonitoring equivalents expert workshop. Regul. Toxicol Pharmacol. 51, S4-S15.

Hendriks, A.J. et al., 2005. Critical body residues linked to octanol-water partitioning, organism composition, and LC50 QSARs: meta-analysis and model. Environ. Sci. Technol. 39, 3226-3236.

Huizer, D. et al., 2012. Separating uncertainty and physiological variability in human PBPK modelling: the example of 2-propanol and its metabolite acetone. Toxicol. Lett. 214, 154-165.

Kawai, T. et al., 1992. Curvi-linear relation between acetone in breathing zone air and acetone in urine among workers exposed to acetone vapor. Toxicol. Lett. 62 85-91.

Kumagai, S., Matsunaga, I., 1995. Physiologically based pharmacokinetic model for acetone. Occup. Environ. Med. 52, 344-352.

Leung, H.W., 1992. Use of physiologically based pharmacokinetic models to establish biological exposure indexes. Am. Ind. Hyg. Assoc. J. 53, 369-374.

Levitzky, M.G., 2003. Ventilation-Perfusion Relationships. Pulmonary Physiology. McGraw-Hill, New York, pp. 113-124.

Martinez, C., Galvan, S., Garcia-Martin, E., Ramos, M.I., Gutierrez-Martin, Y., Agundez, J.A., 2010. Variability in ethanol biodisposition in whites is modulated by polymorphisms in the ADH1B and ADH1C genes. Hepatology 51, 491-500.

Morgan, M.G., Henrion, M., 1990. Uncertainty: A Guide to Dealing with Uncertainty in Quantitative Risk and Policy Analysis. Cambridge University Press, New York.

Morgan, M.S., Schaller, K.H., 1999. An analysis of criteria for biological limit values developed in Germany and in the United States. Int. Arch. Occup. Environ. Health 72, 195-204.

Mork, A.K., Johanson, G., 2006. A human physiological model describing acetone kinetics in blood and breath during various levels of physical exercise. Toxicol Lett. 164, 6-15.

Mork, A.K., Jonsson, F., Johanson, G., 2009. Bayesian population analysis of a washin-washout physiologically based pharmacokinetic model for acetone. Toxicol. Appl. Pharmacol. 240, 423-432.

Nestorov, I., 2001. Modelling and simulation of variability and uncertainty in toxicokinetics and pharmacokinetics. Toxicol. Lett. 120, 411-420.

Pezzagno, G. et al., 1986. Urinary elimination of acetone in experimental and occupational exposure. Scand. J. Work Environ. Health 12, 603-608.

Ragas, A.M., Brouwer, F.P., Buchner, F.L., Hendriks, H.W., Huijbregts, M.A., 2009 Separation of uncertainty and interindividual variability in human exposure modeling. J. Expo. Sci. Environ. Epidemiol. 19, 201-212.

Raman, A. et al., 2004. Water turnover in 458 American adults $40-79$ yr of age. Am. J. Physiol. Renal Physiol. 286, F394-F401.

Safe Work Australia, 2012. Guidance on the Interpretation of Workplace Exposure Standards for Airborne Contaminants. Safe Work Australia.

Satoh, T. et al., 1995. Acetone excretion into urine of workers exposed to acetone in acetate fiber plants. Int. Arch. Occup. Environ. Health 67, 131-134.

Schaller, K.H., Triebig, G., 2002. Acetone [BAT Value Documentation, 1998]. The MAK-Collection for Occupational Health and Safety. Wiley-VCH Verlag GmbH \& Co. KGaA.

Tardif, R. et al., 2002. Impact of human variability on the biological monitoring of exposure to toluene: I. Physiologically based toxicokinetic modelling. Toxicol. Lett. 134, 155-163.

Thomas, R.S. et al., 1996. Variability in biological exposure indices using physiologically based pharmacokinetic modeling and Monte Carlo simulation. Am. Ind. Hyg. Assoc. J. 57, 23-32.

Truchon, G. et al., 2013. Evaluation of occupational exposure: comparison of biological and environmental variabilities using physiologically based toxicokinetic modeling. Int. Arch. Occup. Environ. Health 86, 157-165.

Wang, G. et al., 1994. Blood acetone concentration in "normal people" and in exposed workers $16 \mathrm{~h}$ after the end of the workshift. Int. Arch. Occup. Environ. Health 65, 285-289.

Wigaeus, E. et al., 1981. Exposure to acetone. Uptake and elimination in man. Scand. J. Work Environ. Health 7, 84-94. 